\title{
Mutations in CTC1, encoding conserved telomere maintenance component 1 , cause Coats plus
}

\author{
Beverley H Anderson ${ }^{1,53}$, Paul R Kasher ${ }^{1,53}$, Josephine Mayer ${ }^{1,53}$, Marcin Szynkiewicz ${ }^{1}$, Emma M Jenkinson ${ }^{1}$, \\ Sanjeev S Bhaskar ${ }^{1}$, Jill E Urquhart ${ }^{1}$, Sarah B Daly ${ }^{1}$, Jonathan E Dickerson ${ }^{1}$, James O’Sullivan ${ }^{1}$, \\ Elisabeth Oppliger Leibundgut ${ }^{2}$, Joanne Muter ${ }^{3}$, Ghada M H Abdel-Salem ${ }^{4}$, Riyana Babul-Hirji ${ }^{5}$, Peter Baxter ${ }^{6}$, \\ Andrea Berger ${ }^{7,8}$, Luisa Bonafé9, Janice E Brunstom-Hernandez ${ }^{10,11}$, Johannes A Buckard ${ }^{12}$, David Chitayat ${ }^{5,13}$, \\ Wui K Chong ${ }^{14}$, Duccio M Cordelli ${ }^{15}$, Patrick Ferreira ${ }^{16}$, Joel Fluss ${ }^{17}$, Ewan H Forrest ${ }^{18}$, Emilio Franzoni ${ }^{15}$, \\ Caterina Garone ${ }^{15,19}$, Simon R Hammans ${ }^{20}$, Gunnar Houge ${ }^{21}$, Imelda Hughes ${ }^{22}$, Sebastien Jacquemont ${ }^{23}$, \\ Pierre-Yves Jeannet ${ }^{24}$, Rosalind J Jefferson ${ }^{25}$, Ram Kumar ${ }^{26}$, Georg Kutschke ${ }^{27}$, Staffan Lundberg ${ }^{28}$, \\ Charles M Lourenço ${ }^{29}$, Ramesh Mehta ${ }^{30}$, Sakkubai Naidu ${ }^{31}$, Ken K Nischal ${ }^{32}$, Luís Nunes ${ }^{33}$, Katrin Õunap ${ }^{34}$, \\ Michel Philippart ${ }^{35}$, Prab Prabhakar ${ }^{36}$, Sarah R Risen ${ }^{37}$, Raphael Schiffmann ${ }^{38}$, Calvin Soh ${ }^{39}$, \\ John B P Stephenson ${ }^{40}$, Helen Stewart ${ }^{41}$, Jon Stone ${ }^{42}$, John L Tolmie ${ }^{43}$, Marjo S van der Knaap ${ }^{44}$, Jose P Vieira ${ }^{45}$, \\ Catheline N Vilain ${ }^{46}$, Emma L Wakeling ${ }^{47}$, Vanessa Wermenbol ${ }^{48}$, Andrea Whitney ${ }^{49}$, Simon C Lovell ${ }^{50}$, \\ Stefan Meyer ${ }^{3,51}$, John H Livingston ${ }^{52}$, Gabriela M Baerlocher ${ }^{2}$, Graeme C M Black ${ }^{1}$, Gillian I Rice ${ }^{1}$ \& \\ Yanick J Crow ${ }^{1}$
}

Coats plus is a highly pleiotropic disorder particularly affecting the eye, brain, bone and gastrointestinal tract. Here, we show that Coats plus results from mutations in $C T C 1$, encoding conserved telomere maintenance component 1 , a member of the mammalian homolog of the yeast heterotrimeric CST telomeric capping complex. Consistent with the observation of shortened telomeres in an Arabidopsis CTC1 mutant and the phenotypic overlap of Coats plus with the telomeric maintenance disorders comprising dyskeratosis congenita, we observed shortened telomeres in three individuals with Coats plus and an increase in spontaneous $\gamma \mathrm{H} 2 \mathrm{AX}$-positive cells in cell lines derived from two affected individuals. CTC1 is also a subunit of the $\alpha$-accessory factor (AAF) complex, stimulating the activity of DNA polymerase- $\alpha$ primase, the only enzyme known to initiate DNA replication in eukaryotic cells. Thus, CTC1 may have a function in DNA metabolism that is necessary for but not specific to telomeric integrity.

Telomeres comprise long TTAGGG nucleotide repeats and associated proteins located at the ends of chromosomes. Telomeres both protect the chromosome terminus from unwanted nuclease and DNA repair activities and provide a mechanism to compensate for the inability of DNA polymerase to replicate the $5^{\prime}$ end of a linear chromosome. Telomeric DNA is packaged by a core group of proteins that bind the DNA duplex and the $3^{\prime}$ G overhang on the chromosome terminus. The associated telomere proteins form a protective cap that prevents the chromosome end from eliciting a DNA damage response.
Although it is essential to sequester the DNA end from nuclease and DNA repair activities for much of the cell cycle, during $S$ phase the telomere must be made accessible to telomerase and the DNA replication machinery ${ }^{1}$.

The bone marrow failure syndrome dyskeratosis congenita represents a clinically and genetically heterogeneous collection of phenotypes arising from telomeric protein dysfunction. Dyskeratosis congenita-associated mutations have been identified in the genes encoding DKC1, TERC, TERT, NOP10, NHP2 and TCAB1, which belong to the telomerase holoenzyme responsible for maintaining telomere length, and a member of the shelterin protein complex TINF2, which is responsible for maintaining the structural integrity of the telomere. Thus, dyskeratosis congenita is a disorder of telomere maintenance and is associated with shortened telomeres ${ }^{2,3}$.

Coats plus is a rare disorder of which the most characteristic features are retinal telangiectasia and exudates (Coats disease), a distinctive pattern of intracranial calcification with an associated leukodystrophy and brain cysts, osteopenia with a tendency to fractures and poor bone healing, and a high risk of life-limiting gastrointestinal bleeding and portal hypertension caused by the development of vasculature ectasias in the stomach, small intestine and liver ${ }^{4-9}$ (Fig. 1). These extra-neurological problems differentiate Coats plus from Labrune syndrome (leukoencephalopathy with calcifications and cysts), in which affected individuals otherwise show an identical neuroradiological appearance ${ }^{10}$. Of note, some individuals with Coats plus develop sparse, graying hair, dystrophic nails and a normocytic anemia that might reflect a degree of bone marrow failure. These latter

A full list of author affiliations appears at the end of the paper. 
Figure 1 Features of Coats plus. (a) Cranial axial computed tomography (CT) scan showing characteristic coarse and asymmetrically distributed calcification. Note the cyst in the left frontal cortex. (b) Cranial axial T2 magnetic resonance imaging (MRI) scan taken at similar level to a, highlighting the asymmetric, high signal of the deep and subcortical white matter. (c) $X$ ray of knee illustrating mixed lytic and sclerotic lesions that are mainly metaphyseal. (d) Retinal photograph showing retinal microangiopathy. Scale bar, $\sim 2.5 \mathrm{~mm}$. (e) Endoscopy image showing areas of erythema, which represent vascular ectasia involving the gastric antrum. Scale bar, $\sim 2.5 \mathrm{~cm}$. The neuroradiological features of Labrune syndrome (leukoencephalopathy with calcifications and cysts) are identical to those in $\mathbf{a}$ and $\mathbf{b}$.

features are also observed in dyskeratosis congenita. Furthermore, individuals with Hoyeraal-Hreidarsson or Revesz syndrome, both of which are associated with telomeric shortening, can have intracranial calcification and, in the case of Revesz syndrome, an exudative retinopathy ${ }^{2}$.

In view of reports of both male and female affected siblings, we considered it most probable that Coats plus was an autosomal recessive trait, although we noted that our collection of ten families fulfilling strict diagnostic criteria (characteristic intracranial calcification with white matter changes, exudative retinopathy with telangiectasia and fractures with poor bone healing and/or gastrointestinal vascular ectasia) included only one family in which the parents were known to be related. Because we were unable to define a diseaseassociated locus using SNP arrays (data not shown), we undertook whole-exome sequencing of two siblings (F335_P1 and F335_P2) and two additional unrelated individuals (F332 and F336) showing classical features of Coats plus (Supplementary Table 1). We performed whole-exome capture followed by massively parallel sequencing. Over $4.8 \mathrm{~Gb}$ of sequence was generated for each subject, such that $>76 \%$ of the coding bases of the GENCODE-defined exome were represented by at least ten reads (Supplementary Table 2). We identified single-nucleotide substitutions and small insertion and/or deletion variants using our in-house variant calling pipeline. We analyzed the exome variant profiles under a model of a rare autosomal recessive disorder. Taking into account difficulties in identifying insertion and deletion variants, we looked for genes harboring at least one previously unobserved nonsynonymous or splice-site substitution or a coding insertion or deletion in the same gene in all four individuals, with the same variant(s) required

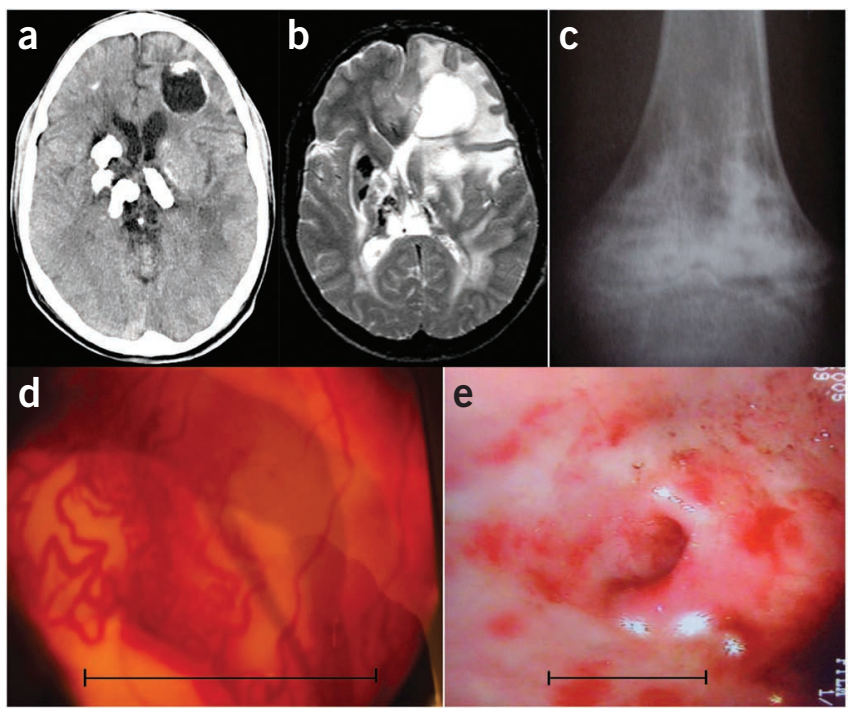

in the siblings examined. CTC1 (encoding conserved telomere maintenance component 1) was highlighted as the only candidate gene when using this strategy. Sanger sequencing confirmed the variants in these affected individuals, and all parents tested showed appropriate heterozygosity for a single variant. In light of these data, we proceeded to sequence an additional nine affected individuals from seven families showing typical disease characteristics.

We identified biallelic CTC1 variants that were likely to be pathogenic in the affected proband(s) from nine of the ten families with Coats plus that were sequenced (Table 1). We also identified two CTC1 variants in a Norwegian individual (F319) having exudative retinopathy with a history of recurrent fractures but in whom cranial imaging had not been undertaken (and who therefore did not fulfill our initial inclusion criteria). In only one family with a prior clinical assignment of Coats plus were we unable to identify CTC1 variants. In the ten families with presumed biallelic mutations, six harbored a nonsense lesion in combination with a missense variant, while the consequences of the splice-site variant seen in F273 and the in-frame deletions observed in F367 and F382 are currently unknown. The probands from F342 carried two missense variants (c.775G $>$ A and c. $2518 \mathrm{C}>\mathrm{T}$ ). Of the 14 distinct

Table 1 Ancestry, pedigree structure and sequence alterations in individuals with Coats plus

\begin{tabular}{|c|c|c|c|c|c|c|}
\hline Family & Ancestry & Tested & Nucleotide alterations & Exons & Amino acid alterations & $\begin{array}{l}\text { Parental } \\
\text { consanguinity }\end{array}$ \\
\hline $\mathrm{F} 273$ & Egyptian & $1 A, M, F$ & Het. c.2831dupC I Het. c. $3011+4 A>C$ & 17। intron 18 & p.His945Serfs*56 I splice donor ${ }^{b}$ & No \\
\hline F319 & Norwegian & $1 \mathrm{~A}$ & Het. c. 721 C>T I Het. c.2923A>G & $5 \mid 17$ & p.GIn241* | p.Arg975Gly & No \\
\hline$F_{33} 2^{a}$ & English & $1 \mathrm{~A}$ & Het. c.724_727delAAAG I Het. c.2959C>T & $5 \mid 18$ & p.Lys242Leufs*41 | p.Arg987Trp & No \\
\hline F335_P1 ${ }^{a}$ & Scottish & $2 \mathrm{~A}$ & Het. c.724_727delAAAG I Het. c.2959C>T & $5 \mid 18$ & p.Lys242Leufs*41 | p.Arg987Trp & No \\
\hline F335_P2a & Scottish & $2 \mathrm{~A}$ & Het. c.724_727delAAAG I Het. c.2959C>T & $5 \mid 18$ & p.Lys242Leufs*41 | p.Arg987Trp & No \\
\hline F336 & English & $1 \mathrm{~A}$ & Het. c.724_727delAAAG I Het. c.2611G>A & $5 \mid 15$ & p.Lys242Leufs*41 | p.Val871Met & No \\
\hline F339 & Canadian & $2 \mathrm{~A}$ & - & - & - & Yes (third cousins) \\
\hline F340 & European-American & $1 \mathrm{~A}$ & Het. c.19C>T I Het. c.2959C>T & 1118 & p.GIn 7* | p.Arg987Trp & No \\
\hline F342_P1 & English and Italian & $2 \mathrm{~A}, \mathrm{M}, \mathrm{F}$ & Het. c. $775 \mathrm{G}>\mathrm{A}$ I Het. c. $2518 \mathrm{C}>\mathrm{T}$ & $5 \mid 15$ & p.Val259Met I p.Arg840Trp & No \\
\hline F342_P2 & English and Italian & $2 A, M, F$ & Het. c. $775 \mathrm{G}>$ A I Het. c. $2518 \mathrm{C}>\mathrm{T}$ & $5 \mid 15$ & p.Val259Met I p.Arg840Trp & No \\
\hline F345 & Swiss and French & $1 \mathrm{~A}$ & Het. c.724_727delAAAG I Het. c.1507G>C & $5 \mid 9$ & p.Lys242Leufs*41 | p.Gly503Arg & No \\
\hline F367 & African and European & $1 \mathrm{~A}, 1 \mathrm{U}, \mathrm{M}$ & Het. c.859C>T I Het. c.2954_2956delGTT & $6 \mid 18$ & p.Arg287* | p.Cys985del & No \\
\hline F382 & Portuguese & $1 \mathrm{~A}, 1 \mathrm{U}, \mathrm{M}, \mathrm{F}$ & $\begin{array}{l}\text { Het. c.2954_2956delGTT I } \\
\text { Het. c.3586_3606del }\end{array}$ & $18 \mid 23$ & $\begin{array}{l}\text { p.Cys985del I p.Leu1196_ } \\
\text { Arg1202del }\end{array}$ & No \\
\hline
\end{tabular}


Figure 2 Schematic representation of the human CTC1 gene. (a) CTC1 spans 23,273 bp of genomic sequence on chromosome 17p13.1 $(8,128,140-8,151,413)$. Neighboring genes are also shown. Tel., telomere; cen., centromere. (b) Position of identified mutations within the CTC1 gene. Protein alterations are given in brackets.

mutations observed, three were seen in more than one family (c.724_727del, four families; c.2954_2956del, two families; c.2959C>T, three families) (Table 1).

Reflecting apparently rapid evolutionary divergence, some telomeric proteins show poor interspecies conservation ${ }^{11}$. The human CTC1 protein sequence shares only $69 \%$ identity with mouse, $30 \%$ with zebrafish and $14 \%$ with Arabidopsis. Not unexpectedly, although the residues affected by nonsynonymous missense changes are well conserved across mammals, ClustalW alignment showed a relatively low level of residue conservation in other species (Supplementary Fig. 1). Related to this, 15 of the 20 CTC1 mutations we observed occurred in one of four exons

(Fig. 2). However, although we suspect that this clustering is of functional significance, it is difficult to model the domain structure of CTC1 because of the high degree of sequence divergence from other proteins ${ }^{12}$. Of note, none of the 14 distinct, putatively pathogenic CTC1 variants have been annotated as polymorphisms in dbSNP. Moreover, 13 of these variants were not seen in 1,730 EuropeanAmerican and African-American subjects collated in the Exome Variant Server database, and the c.19C > T transition seen in F340 was recorded in only 1 of 1,497 European-Americans (the same ancestral background as the affected proband).

Cerebroretinal microangiopathy with calcifications and cysts (CRMCC) is a term coined to encompass the Coats plus and Labrune syndrome phenotypes, on the grounds that the neuroradiological characteristics of these two disorders are essentially identical ${ }^{7,8}$. Thus, we sequenced CTC1 in a collection of probands from 21 families showing stereotypical intracranial calcification and white matter changes in the absence of extra-neurological features, but we were unable to identify any likely pathogenic variants (Supplementary Table 3).

Recently, it has been shown that CTC1 interacts with STN1 and TEN1 to form the mammalian homolog of the yeast heterotrimeric Cdc13-Stn1-Ten1 (CST) telomeric capping complex ${ }^{13,14}$. Consequently, we also undertook Sanger sequencing of OBFC1 (STN1) and TEN1 in our collection of individuals with Labrune syndrome and in the proband with Coats plus in whom we identified no CTC1 mutations. Again, sequencing identified no variants of likely pathogenic significance (Supplementary Tables 3 and 4 ).

In view of the finding of shortened telomeres in an Arabidopsis CTC1 mutant $^{13}$ and the phenotypic overlap between Coats plus and the telomeric maintenance disorders comprising dyskeratosis congenita, we measured telomere lengths ${ }^{15}$ in F332, F367 and F382 at the ages of 20, 20 and 3 years, respectively. Samples were also available from the mother and heterozygous brother of F367 and from both parents of F332 and F382. Testing showed markedly shortened telomeres in lymphocytes and granulocytes from the peripheral blood of F367 and F382 and telomeric lengths at the lower range of normal in the heterozygous relatives of these individuals (Fig. 3). In F332, telomere length was recorded on the first percentile for age in both lymphocytes and granulocytes, with parental telomere lengths also at the lower range of normal.

Considering the enrichment for CTC1 variants in individuals with a predefined clinical and radiological diagnosis, we are confident that mutations in CTC1 represent the major cause of Coats plus. The observation of a single family, F339, in whom we were unable to identify mutations suggests possible genetic heterogeneity or a phenocopy. Of note, the parents of this child are related as third cousins, but there was no evidence of homozygosity around any of the three CST protein-encoding genes (data not shown).

Although Coats plus is rare and inherited as an autosomal recessive trait, none of the ten mutation-positive families we identified are consanguineous. In keeping with this, all of the affected individuals in these families are compound heterozygotes for two different CTC1 variants (with six of ten families harboring a nonsense and missense mutation in combination). This observation helps to explain our inability to define a disease locus using autozygosity mapping and leads us to speculate that biallelic null mutations might be incompatible with development, whereas homozygosity for (most) missense variants may be associated with a normal phenotype or a different pathogenic one.

We and others have noted the highly stereotyped neuroradiological features common to Coats plus and Labrune syndrome, leading to the introduction of the umbrella term CRMCC. However, a recent analysis of our cohort (data not shown) suggested that the two groups might be distinguished according to the presence or absence of extra-neurological features, as, in our experience, individuals without retinal abnormalities have never shown any skeletal or gastrointestinal manifestations over extended time periods. The genetic data presented here support this conclusion, indicating that Coats plus and Labrune syndrome are not allelic.

CTC1 comprises 23 exons and encodes a 134.5-kDa protein. Because of rapid evolutionary divergence, the human CST complex was only defined recently ${ }^{13,14}$. This led to the recognition that mammalian 

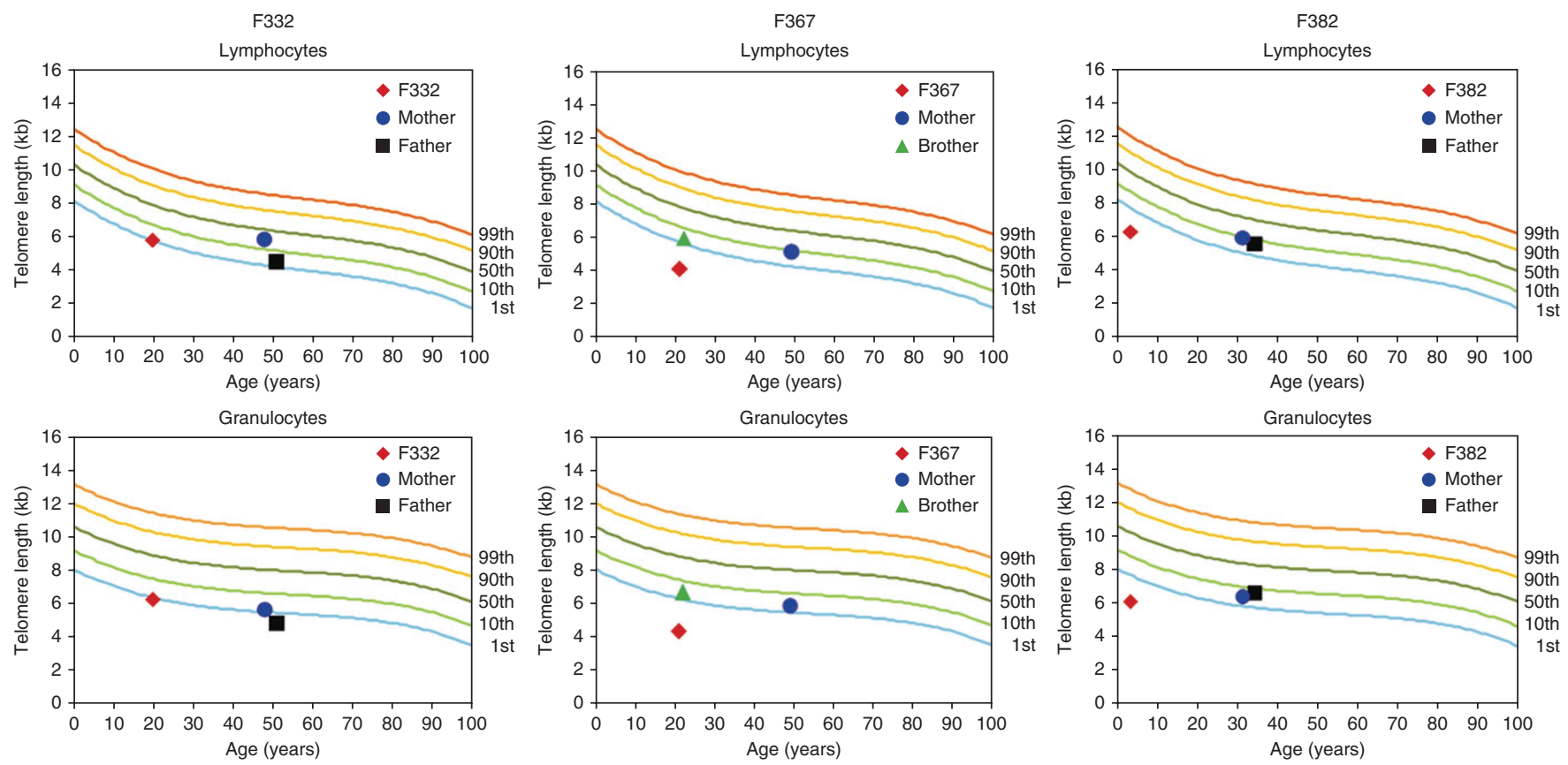

Figure 3 Telomere length analysis in subsets of leukocytes as measured by automated multicolor flow-FISH. Telomere lengths were assessed in lymphocytes and granulocytes from F332, F367 and F382 at the ages of 20, 20 and 3 years, respectively, and from unaffected family members harboring a single CTC1 mutation. Telomere lengths were markedly shortened in two affected individuals (F367 and F382) and were at the lower range of normal in their heterozygous relatives. In F332, telomere length was recorded on the first percentile for age in both lymphocytes and granulocytes, with parental telomere lengths at the lower range of normal (being less than the first percentile in the father in granulocytes). The reference range for telomere length over age in percentiles was derived from telomere length analyses in lymphocytes and granulocytes from 400 healthy individuals.

CTC1 is identical to one subunit of $\alpha$-accessory factor (AAF-132), whereas a second subunit of AAF (AAF-44, also known as OBFC1) corresponds to mammalian STN1 (ref. 16).

The AAF complex stimulates the activity of DNA polymerase- $\alpha$ primase, the only enzyme known to initiate DNA replication in eukaryotic cells ${ }^{17}$. AAF functions by binding single-stranded DNA (ssDNA) and enhancing DNA polymerase- $\alpha$ primase association with a DNA template. The finding that human CTC1-STN1 modulates DNA polymerase- $\alpha$ primase activity indicates that, as for budding yeast CST, mammalian CTC1-STN1 provides a link to the lagging strand replication machinery. Thus, it can be proposed that a conserved function of CST is to promote efficient priming of telomeric $\mathrm{C}$-strand synthesis. In addition, given the role of Cdc13 in budding yeast, another conserved function of the CST complex could be to regulate telomerase. All of these processes may contribute to maintaining telomeric DNA integrity and could couple the conventional replication process to telomere-specific priming and telomerasedependent elongation steps ${ }^{18}$ (see Supplementary Fig. 2).

Experiments in budding yeast and Arabidopsis have identified a functional role for the CST complex in maintaining telomeric structural integrity, and our finding of shortened telomeres in individuals with CTC1 mutations confirms an important role for the CST complex in mammalian telomere metabolism. However, the CST complex only partially localizes to telomeres and binds to ssDNA in a sequence-independent manner (through the oligonucleotide/oligosaccharide-binding (OB) fold domains predicted to be present in all three subunits $)^{13,14}$. Of note, knockdown of CTC1 in human cells resulted in an increase in the number of $\gamma \mathrm{H} 2 \mathrm{AX}$ foci not confined to telomeres ${ }^{13}$. Although we did not study chromosomal localization, we observed a significant increase in spontaneous $\gamma \mathrm{H} 2 \mathrm{AX}$-positive cells in cell lines from the two affected individuals (F332 and F382) that we were able to examine $(P=0.0001$ and 0.0007 , respec- tively), indicative of an ongoing DNA damage response (Supplementary Fig. 3). Consequently, it is possible that the CST complex may have a more general role in DNA replication and repair that is frequently required by but not specific to telomeres.

Our interest in Coats plus and Labrune syndrome derives from ongoing research into disorders associated with the presence of intracranial calcification. As such, our cohort is biased toward individuals with obvious neurological involvement. It is possible then that the phenotypic spectrum associated with CTC1 mutations may be broader than that presented here. Of note, phenotypic overlap with dyskeratosis congenita was appreciated at the time of the first clinical description of Coats plus, leading us to speculate that cohorts of individuals with a diagnosis of dyskeratosis congenita may be enriched for pathogenic variants in CTC1, OBFC1 (STN1) and/or TEN1.

URLs. National Heart, Lung, and Blood Institute (NHLBI) Exome Sequencing Project (ESP) Exome Variant Server, http://evs.gs.washington. edu/EVS/; SplicePort, http://spliceport.cs.umd.edu/; GENCODE, http://www.gencodegenes.org/; ClustalW, http://www.ebi.ac.uk/tools/ msa/clustalw2/; Primer3Plus, http://www.primer3plus.com/.

\section{METHODS}

Methods and any associated references are available in the online version of the paper at http://www.nature.com/naturegenetics/.

Accession codes. Nucleotide sequences are available from Entrez PubMed for human CTC1 (NM_025099.5), human OBFC1 (STN1) (NM_024928.4) and human TEN1 (NM_001113324.2), as is the human CTC1 protein sequence (NP_079375.3).

Note: Supplementary information is available on the Nature Genetics website. 


\section{ACKNOWLEDGMENTS}

We are very grateful to the affected families for their involvement in our research. Y.J.C. acknowledges the Manchester National Institute for Health Research (NIHR) Biomedical Research Centre. This work has received support from the Great Ormond Street Hospital Children's Charity and from the Newlife Foundation. We thank the NHLBI Grand Opportunity (GO) Exome Sequencing Project and acknowledge its ongoing studies that produced and provided exome variant calls for comparison, including the Lung GO Sequencing Project (HL-102923), the Women's Health Initiative (WHI) Sequencing Project (HL-102924), the Broad GO Sequencing Project (HL-102925), the Seattle GO Sequencing Project (HL-102926) and the Heart GO Sequencing Project (HL-103010). We also thank G. Forte for organizing courier delivery of patient materials.

\section{AUTHOR CONTRIBUTIONS}

B.H.A. and J.O. performed exome sequencing. B.H.A., M.S., G.I.R. and E.M.J. were responsible for Sanger sequencing. J. Mayer, S.S.B. and J.E.D. undertook analysis of the exome sequence data. J.E.U. and S.B.D. performed SNP analyses. J.H.L. was responsible for neuroradiological phenotyping. G.M.B. and E.O.L. performed the telomeric length analysis. S.M. and J. Muter assessed $\gamma \mathrm{H} 2 \mathrm{AX}$ positivity. S.C.L. analyzed CTC1 structural domains. G.I.R. and G.C.M.B. provided critical input into project development and manuscript preparation. Y.J.C. designed and supervised the project and wrote the manuscript with the support of P.R.K. All other authors identified subjects with Coats plus and/or Labrune syndrome and performed related clinical and laboratory studies. Phenotypic overlap with dyskeratosis congenita was originally appreciated by J.L.T. and J.B.P.S. at the time of the first clinical description of Coats plus.

\section{COMPETING FINANCIAL INTERESTS}

The authors declare no competing financial interests.

Published online at http://www.nature.com/naturegenetics/.

Reprints and permissions information is available online at http://www.nature.com/ reprints/index.html.

1. Jain, D. \& Cooper, J.P. Telomeric strategies: means to an end. Annu. Rev. Genet. 44, 243-269 (2010)

2. Savage, S.A. \& Bertuch, A.A. The genetics and clinical manifestations of telomere biology disorders. Genet. Med. 12, 753-764 (2010)
3. Nelson, N.D. \& Bertuch, A.A. Dyskeratosis congenita as a disorder of telomere maintenance. Mutat. Res. published online, doi:10.1016/j.mrfmmm.2011.06.008 (2 July 2011).

4. Tolmie, J.L., Browne, B.H., McGettrick, P.M. \& Stephenson, J.B. A familial syndrome with coats' reaction retinal angiomas, hair and nail defects and intracranial calcification. Eye (Lond.) 2, 297-303 (1988).

5. Crow, Y.J. et al. Coats' plus: a progressive familial syndrome of bilateral Coats' disease, characteristic cerebral calcification, leukoencephalopathy, slow pre- and post-natal linear growth and defects of bone marrow and integument. Neuropediatrics 35, 10-19 (2004)

6. Nagae-Poetscher, L.M. et al. Leukoencephalopathy, cerebral calcifications, and cysts: new observations. Neurology 62, 1206-1209 (2004).

7. Linnankivi, T. et al. Cerebroretinal microangiopathy with calcifications and cysts. Neurology 67, 1437-1443 (2006)

8. Briggs, T.A. et al. Cerebroretinal microangiopathy with calcifications and cysts (CRMCC). Am. J. Med. Genet. A. 146A, 182-190 (2008).

9. Briggs, T.A. et al. Treatment of gastrointestinal bleeding in a probable case of cerebroretinal microangiopathy with calcifications and cysts. Mol. Syndromol. 1, 159-162 (2011).

10. Labrune, P. et al. Extensive brain calcifications, leukodystrophy, and formation of parenchymal cysts: a new progressive disorder due to diffuse cerebral microangiopathy. Neurology 46, 1297-1301 (1996).

11. Linger, B.R. \& Price, C.M. Conservation of telomere protein complexes: shuffling through evolution. Crit. Rev. Biochem. Mol. Biol. 44, 434-446 (2009).

12. Price, C.M. et al. Evolution of CST function in telomere maintenance. Cell Cycle 9, 3157-3165 (2010).

13. Surovtseva, Y.V. et al. Conserved telomere maintenance component 1 interacts with STN1 and maintains chromosome ends in higher eukaryotes. Mol. Cel/ 36, 207-218 (2009).

14. Miyake, Y. et al. RPA-like mammalian Ctc1-Stn1-Ten1 complex binds to singlestranded DNA and protects telomeres independently of the Pot1 pathway. Mol. Cell 36, 193-206 (2009)

15. Baerlocher, G.M., Vulto, I., de Jong, G. \& Lansdorp, P.M. Flow cytometry and FISH to measure the average length of telomeres (flow FISH). Nat. Protoc. 1, 2365-2376 (2006)

16. Casteel, D.E. et al. A DNA polymerase- $\alpha$-primase cofactor with homology to replication protein A-32 regulates DNA replication in mammalian cells. J. Biol. Chem. 284, 5807-5818 (2009).

17. Goulian, M. \& Heard, C.J. The mechanism of action of an accessory protein for DNA polymerase $\alpha /$ primase. J. Biol. Chem. 265, 13231-13239 (1990).

18. Giraud-Panis, M.J., Teixeira, M.T., Geli, V. \& Gilson, E. CST meets shelterin to keep telomeres in check. Mol. Cell 39, 665-676 (2010).

${ }^{1}$ Manchester Academic Health Science Centre, University of Manchester, Genetic Medicine, Manchester, UK. ${ }^{2}$ Experimental Hematology, Department of Clinical Research, University Hospital and University of Bern, Bern, Switzerland. ${ }^{3}$ Stem Cell and Leukaemia Proteomics Laboratory, University of Manchester, Manchester, UK. ${ }^{4}$ Clinical Genetics Department, Human Genetics and Genome Research Division, National Research Centre, Cairo, Egypt. ${ }^{5}$ Division of Clinical and Metabolic Genetics, The Hospital for Sick Children, University of Toronto, Toronto, Canada. ${ }^{6}$ Ryegate Centre, Sheffield Children's National Health Service (NHS) Foundation Trust, Sheffield, UK. ${ }^{7}$ Department of Pediatric Neurology, University of Mainz, Mainz, Germany. ${ }^{8}$ Department of Pediatric Neurology, Children's Hospital Harlaching, Munich, Germany. ${ }^{9}$ Division of Molecular Pediatrics, Centre Hospitalier Universitaire Vaudois, Lausanne, Switzerland. ${ }^{10}$ Department of Neurology, Washington University School of Medicine, St. Louis Children's Hospital, Saint Louis, Missouri, USA. ${ }^{11}$ Department of Pediatrics, Washington University School of Medicine, St. Louis Children's Hospital, Saint Louis, Missouri, USA. ${ }^{12}$ Ärztlicher Leiter, Sozialpädiatrisches Zentrum am Evangelisches Krankenhaus (EVK) Düsseldorf, Düsseldorf, Germany. ${ }^{13}$ Department of Obstetrics and Gynecology, The Prenatal Diagnosis and Medical Genetics Program, Mount Sinai Hospital, University of Toronto, Toronto, Canada. ${ }^{14}$ Department of Radiology, Great Ormond Street Hospital for Children NHS Trust, London, UK. ${ }^{15}$ Child Neuropsychiatry Unit, University of Bologna, S. Orsola-Malpighi Hospital, Bologna, Italy. ${ }^{16}$ Division of Medical Genetics, Alberta Children's Hospital, Calgary, Canada. ${ }^{17}$ Pediatric Neurology, Geneva Children's Hospital, Geneva, Switzerland. ${ }^{18}$ Glasgow Royal Infirmary, Glasgow, UK. ${ }^{19}$ Department of Neurology, Columbia University Medical Center, New York, New York, USA. ${ }^{20}$ Wessex Neurological Centre, Southampton General Hospital, Southampton, UK. ${ }^{21}$ Center for Medical Genetics and Molecular Medicine, Haukeland University Hospital, Bergen, Norway. ${ }^{22}$ Royal Manchester Children's Hospital, Central Manchester University Hospitals NHS Foundation Trust, Manchester, UK. ${ }^{23}$ Service de Génétique Médicale, Centre Hospitalier Universitaire Vaudois, Lausanne, Switzerland. ${ }^{24}$ Pediatric Neurology Unit, Department of Pediatrics, Centre Hospitalier Universitaire Vaudois, Lausanne, Switzerland. ${ }^{25}$ Dingley Specialist Children's Centre, Royal Berkshire Hospital, Reading, UK. ${ }^{26}$ Department of Neurology, Alder Hey Children's NHS Foundation Trust, Liverpool, UK. ${ }^{27}$ Klinik für Allgemeine Pädiatrie und Neonatologie, Kliniken für Kinder- und Jugendmedizin, Universitätsklinikum des Saarlandes, Homburg, Germany. ${ }^{28}$ Department of Women's and Children's Health, Uppsala University, Uppsala, Sweden. ${ }^{29}$ Neurogenetics Unit, Department of Neuroscience, Faculdade de Medicina de Ribeirão Preto, University of Sao Paulo, Sao Paulo, Brazil. ${ }^{30}$ Bedford Hospital NHS Trust, Bedford, UK. ${ }^{31}$ Neurogenetics Unit, Hugo Moser Research Institute, Kennedy Krieger Institute, Johns Hopkins University School of Medicine, Baltimore, Maryland, USA. 32University of Pittsburgh Medical Center (UPMC) Children's Hospital of Pittsburgh and Eye Center, Pittsburgh, Pennsylvania, USA. ${ }^{33}$ Department of Medical Genetics, Hospital Dona Estefânia Centro Hospitalar de Lisboa Central (CHLC), Faculdade de Ciências Médicas de Lisboa Universidade Nova de Lisboa (UNL), Lisbon, Portugal. ${ }^{34}$ Department of Genetics, United Laboratories, Tartu University Hospital, Tartu, Estonia. ${ }^{35}$ Brain Research Institute, David Geffen School of Medicine, University of California, Los Angeles, Los Angeles, California, USA. ${ }^{36}$ Department of Neurology, Great Ormond Street Hospital for Children, London, UK. ${ }^{37}$ Department of Neurology and Developmental Medicine, Kennedy Krieger Institute, Johns Hopkins University School of Medicine, Baltimore, Maryland, USA. ${ }^{38}$ Institute of Metabolic Disease, Baylor Research Institute, Dallas, Texas, USA. ${ }^{39}$ Neuroradiology Department, Salford Royal NHS Foundation Trust, Salford, UK. ${ }^{40}$ Fraser of Allander Neurosciences Unit, Royal Hospital for Sick Children, Glasgow, UK. ${ }^{41}$ Clinical Genetics Department, Oxford Radcliffe Hospitals NHS Trust, Oxford, UK. ${ }^{42}$ Department of Clinical Neurosciences, Western General Hospital, Edinburgh, UK. ${ }^{43}$ Department of Clinical Genetics, Royal Hospital for Sick Children, Yorkhill, Glasgow, UK. ${ }^{44}$ Department of Child Neurology, VU University Medical Center, Amsterdam, The Netherlands. ${ }^{45}$ Department of Neurology, Hospital Dona Estefânia, Centro Hospitalar de Lisboa Central, Lisbon, Portugal. ${ }^{46}$ Department of Medical Genetics, Université Libre de Bruxelles (ULB) Center of Human Genetics, Université Libre de Bruxelles, Brussels, Belgium. ${ }^{47}$ North West Thames Regional Genetics Service, North West London Hospitals NHS Trust, Harrow, UK. ${ }^{48}$ Department of Pediatric Neurology, Université Libre de Bruxelles, Hôpital Erasme, Brussels, Belgium. ${ }^{49}$ Southampton University Hospital Foundation Trust, Southampton, UK. ${ }^{50}$ Faculty of Life Sciences, University of Manchester, Manchester, UK. ${ }^{51}$ Paediatric Oncology, Central Manchester University Hospitals NHS Foundation Trust, Manchester, UK. ${ }^{52}$ Department of Paediatric Neurology, Leeds General Infirmary, Leeds, UK. ${ }^{53}$ These authors contributed equally to this work. Correspondence should be addressed to Y.J.C. (yanickcrow@mac.com). 


\section{ONLINE METHODS}

Subject ascertainment. Subjects were ascertained internationally through colleagues in pediatric neurology and clinical genetics. Written informed consent was obtained for all participants. The study had full ethical approval from the Leeds Multicentre Research Ethics Committee (07/Q1206/7).

Exome sequencing. Genomic DNA was extracted from lymphocytes from affected individuals, parents and siblings by standard techniques. For wholeexome analysis, targeted enrichment and sequencing were performed on DNA extracted from peripheral blood from four individuals (F332, F335_P1, F335_P2 and F336). Enrichment was performed using the SureSelect Human All Exon Kit v.1 (Agilent) for the Applied Biosystems SOLiD system. Emulsion PCR (ePCR) was conducted on the resultant sample library, and products were then sequenced on a SOLiD 4 sequencer (Life Technologies). Sequence data were mapped using SOLiD Bioscope software (Life Technologies), with the hg18 human genome as a reference. SNPs were called using the diBayes tool in the BioScope software suite with the medium stringency setting and then filtered to remove those SNPs with less than $5 \times$ coverage. A total of $4.8 \mathrm{~Gb}$ of sequence mapped uniquely to the hg18 genome reference, with $76.4 \%$ of the targeted exome covered at tenfold or higher (Supplementary Table 2).

Sanger sequencing. Mutation analysis was performed by direct sequencing of purified genomic PCR products using the BigDye Terminator v3.1 cycle sequencer system (Applied Biosystems). Primers were designed for individual exons and intron boundaries of CTC1, OBFC1 (STN1) and TEN1 using
Primer3Plus and the reference sequences of each gene (primer sequences are provided in Supplementary Table 5; details of experimental conditions are available on request).

Telomere length measurement. Telomere length was measured in white blood cell subsets by automated multicolor flow-FISH analysis ${ }^{15}$ of granulocytes, CD45RA ${ }^{+}$lymphocytes (naive T cells), CD45RA- lymphocytes (memory T cells), CD $20^{+}$lymphocytes (B cells), CD $57^{+}$lymphocytes (NK/NKT cells) and total leukocytes, and compared with age-matched controls. 'Markedly short' was defined as less than the first percentile for age.

FACS for measurement of $\gamma \mathrm{H} 2 \mathrm{AX}$ positivity. Lymphoblastoid cells from two affected individuals (F332 and F382) and three control individuals were seeded at a density of $5 \times 10^{5}$ cells per well of a six-well plate (Sigma) and maintained at $37^{\circ} \mathrm{C}$ overnight. Cells were pelleted and washed in PBS and fixed in $500 \mu \mathrm{l}$ of $1 \times$ Fixation Solution (Millipore $\gamma \mathrm{H} 2 \mathrm{AX}$ Phosphorylation Assay Kit) for $20 \mathrm{~min}$ at $4{ }^{\circ} \mathrm{C}$. Cells were pelleted and washed in $1 \mathrm{ml}$ of PBS, pelleted again and resuspended in $50 \mu \mathrm{l}$ of $1 \times$ permeabilizing solution (Millipore $\gamma \mathrm{H} 2 \mathrm{AX}$ Phosphorylation Assay Kit), and $3.5 \mu \mathrm{l}$ of appropriate antibody was added (FITC-conjugated antibody to $\gamma \mathrm{H} 2 \mathrm{AX}$ or control FITC-conjugated rabbit IgG) and incubated at $4{ }^{\circ} \mathrm{C}$ for $20 \mathrm{~min}$. We added $100 \mu \mathrm{l}$ of $1 \times$ washing solution to each sample, and cells were pelleted and resuspended in $400 \mu \mathrm{l}$ of PBS. Samples were analyzed using the BD Biosciences FACSCalibur using CellQuest Pro software, and data were analyzed using FlowJo. 
Copyright of Nature Genetics is the property of Nature Publishing Group and its content may not be copied or emailed to multiple sites or posted to a listserv without the copyright holder's express written permission.

However, users may print, download, or email articles for individual use. 